\title{
Aproximación conceptual de las competencias en el contexto educativo*
}

\author{
Fernando Guío Gutiérrez** \\ Recibido: octubre 3 de 2016 • Evaluado: octubre 13 de 2016
}

Aceptado: octubre 27 de 2016

Resumen

El concepto de competencias se ha venido trasladando del ámbito empresarial a las instituciones educativas, fundamentalmente hacia la educación superior, que busca procesos educativos de calidad en oposición a los modelos establecidos tradicionalmente fundamentados en la transmisión de la información, centradas en el docente y su conocimiento, con poca intervención activa de los estudiantes. Sin embargo, en el entorno académico se han establecido una serie de conceptos sobre las competencias que generan comprensiones equivocadas del término. En este sentido, se propone una aproximación conceptual y algunas reflexiones sobre el desarrollo del enfoque basado en competencias en la institución educativa.

Palabras clave: competencia, competencias docentes, enfoque basado en competencias.

* Artículo de reflexión derivado del seminario fundamentos de la educación basada en competencias orientado por la Dra. Margarita Alemán Vargas: Centro Universitario Mar de Cortés, octubre 2016.

"Magister en Pedagogía Universidad de La Sabana. Doctorante Educación Basada en Competencias, Centro Universitario Mar de Cortés. Especialista en Educación. Licenciado en Educación Física Universidad Pedagógica Nacional. Docente Facultad de Cultura Física Universidad Santo Tomás. Docente investigador Universidad de La Sabana. Docente Educación Física Colegio Agustín Fernández IED. 


\section{Conceptual approach of the competences in the educational context}

Abstract

The concept of competences has been transferred from the business field to educational institutions, mainly towards higher education, which seeks quality educational processes in opposition to the traditionally established models based on the transmission of information, focused on the teacher and his knowledge, with little active student participation. However, in the academic environment, a series of concepts about competences have been established that generate misunderstandings of the term. In this sense, we propose a conceptual approach and some reflections on the development of the competency-based approach in the educational institution.

Keywords: Competence, teaching competencies, competence-based approach. 


\section{Introducción}

La educación basada en competencias constituye un enfoque pedagógico que se está posicionando en muchas de las instituciones como una alternativa para el mejoramiento de la calidad educativa y como respuesta a la necesidad de abordar procesos situados, contextualizados y pertinentes que la sociedad de hoy demanda de la educación y que se opone a los esquemas tradicionales de una enseñanza basada en la repetición y la memoria. En el ámbito académico se ha venido vinculando el término de competencia, planteado inicialmente para el ámbito laboral, incursiona primero en la educación superior y se ha extendido a otros niveles educativos, incluso en el preescolar, haciendo que en el debate pedagógico se interprete de manera polisémica su concepto.

Es así como se exponen algunos conceptos generales de la competencia, que si bien, responden de manera acertada a algunas de las expectativas educativas y a los desempeños esperados para los estudiantes, no son lo suficientemente sólidos, dificultando su compresión. Sin embargo, es posible encontrar constructos más elaborados y orientadores para las prácticas didácticas de los docentes.

Como las múltiples maneras de presentar el abordaje conceptual de la competencia, evidencian una dispersión de definiciones, se hace necesario establecer un consenso que permita orientar de una mejor manera su interpretación y por ende sus didácticas en la escuela. En este sentido, el documento, más que proponer una nueva definición, pretende exponer una aproximación conceptual fundamentada en los constructos de los autores, para facilitar en los docentes la interpretación de su significado, sus componentes y sus características para una implementación real de la competencia en la escuela y reflexionando sobre las mismas competencias que debe apropiar el docente en su ejercicio pedagógico.

\section{Aproximación conceptual de la competencia en el ámbito educativo}

Haciendo referencia al ámbito educativo, algunos autores señalan que el termino competencia es utilizado por primera vez en la lingüística por 
Chomsky en 1965, donde se define a la manera de apropiarse y utilizar el lenguaje como competencia lingüística. (Barón Birchenall, 2014). Es así, como a partir de Chomsky, se genera la reflexión sobre como aprenden las personas y como se apropian de ese saber. En adelante surgen académicos y organizaciones que le otorgan significados desde diferentes perspectivas.

Los primeros conceptos de competencia se refieren a aquello que caracteriza a una persona capaz de realizar una tarea concreta de manera eficiente (Zabala, \& Arnau, 2008). Es decir, el saber hacer en un contexto. En el Proyecto Tuning América Latina (2007), se presentan las competencias como capacidades complejas integradas que la educación debe formar en los individuos para que puedan desempeñarse como sujetos responsables en diferentes situaciones y contextos de la vida social y personal, y les permita ver, hacer, actuar y disfrutar.

En otro sentido, y más cercano a la función social de la educación, Tobón (2008) señala que las competencias no son un modelo pedagógico, sino un enfoque para la educación, que puede llevarse a cabo desde cualquiera de los modelos pedagógicos existentes o desde la integración de varios de ellos. Sugiere, además, que antes de adoptar este enfoque se debe construir en consenso y de manera participativa el modelo pedagógico de la institución educativa. Una vez aclarados estos aspectos, define la competencia como:

Un proceso complejo de desempeño con idoneidad en determinados contextos, integrando diferentes saberes (saber ser, saber hacer, saber conocer y saber convivir), para realizar actividades y/o resolver problemas con sentido de reto, motivación, flexibilidad, creatividad, comprensión y emprendimiento, dentro de una perspectiva de procesamiento metacognitivo, mejoramiento continuo y compromiso ético, con la meta de contribuir al desarrollo personal, la construcción y afianzamiento del tejido social, la búsqueda continua del desarrollo económico-empresarial sostenible, y el cuidado y protección del ambiente y de las especies vivas (Tobón, 2008, p. 5).

De la misma manera el concepto de competencia se define en función del enfoque pedagógico que se pretende desarrollar, por ejemplo, el enfoque conductista les otorga gran importancia a los desempeños y por ello las competencias se construyen a partir de comportamientos observables. En el 
enfoque funcionalista se fundamenta en el trabajo de campo, la exploración y la vivencia en contravía a la sumisión y obediencia del conductismo. En el enfoque cognitivo, se vinculan los procesos de pensamiento que utilizan las personas para realizar tareas, adquirir conocimientos y mejorar los desempeños. Zabala y Arnou (2008) muestran cómo varios autores intentan desde varios enfoques definir la competencia:

Tabla 1. Tendencias en la definición de las competencias

\begin{tabular}{|c|c|c|c|}
\hline $\begin{array}{l}\text { Componente } \\
\text { fundamental }\end{array}$ & Objetivo & $\begin{array}{l}\text { Condición } \\
\text { de calidad }\end{array}$ & $\begin{array}{l}\text { Escenario de } \\
\text { intervención }\end{array}$ \\
\hline $\begin{array}{l}\text { - Características } \\
\text { - Capacidades } \\
\text { - Conocimientos } \\
\text { - Actitudes } \\
\text { - Destrezas } \\
\text { - Habilidades } \\
\text { - Cualidades } \\
\text { - Aptitudes } \\
\text { - Comportamientos } \\
\text { - Inteligencia }\end{array}$ & $\begin{array}{l}\text { - Rendimiento } \\
\text { - Ejecución de } \\
\text { tareas } \\
\text { - Desempeños } \\
\text { - Decisiones } \\
\text { - Resolución de } \\
\text { problemas }\end{array}$ & $\begin{array}{l}\text { - Eficiencia } \\
\text { - Efectividad } \\
\text { - Eficacia } \\
\text { - Éxito } \\
\text { - Movilización } \\
\text { de recursos }\end{array}$ & $\begin{array}{l}\text { - Formación } \\
\text { profesional } \\
\text { - Escuela } \\
\text { - Trabajo } \\
\text { - Grupos de } \\
\text { situaciones }\end{array}$ \\
\hline
\end{tabular}

Fuente: Zabala y Arnou (2008).

No obstante la diversidad de propuestas es posible encontrar aspectos en común en las definiciones, como la búsqueda de la innovación, mejorar los conocimientos, la calidad educativa y las interacciones entre la teoría y la práctica. En este sentido se propone como una aproximación conceptual holística, comprensiva y más cercana a las intenciones educativas de los maestros, la siguiente afirmación: la competencia es la integración y movilización de saberes hacia escenarios reales de actuación que genera respuestas idóneas, responsables y creativas a diferentes situaciones y contextos, y contribuyen al desarrollo personal y social. 


\section{Características y componentes de la competencia}

A partir de la aproximación conceptual propuesta, se puede inferir cuales son las características o condiciones que debería presentar una competencia:

La integración y movilización de saberes: Toda competencia debe incluir dimensiones del ser, hacer, saber/aprender, comunicar, convivir y además generar la posibilidad de trasladar esas dimensiones o saberes a las actuaciones de las personas.

Escenarios reales de actuación: La competencia se hace evidente en la realidad, cuando la persona se enfrenta a situaciones reales y debe tomar decisiones inteligentes y acciones apropiadas. El desempeño en una competencia no es hipotético y en este sentido la escuela debería fundamentar sus prácticas no en sesiones de clase, sino más bien, en experiencias educativas.

Respuestas idóneas, responsables y creativas: La competencia debe comprometer al comportamiento ético, tolerante y responsable de las personas, quienes, no solamente deben buscar un desempeño ideal y eficiente, sino también respuestas creativas e innovadoras en sus actuaciones y compromisos.

Diferentes situaciones y contextos: La competencia les permite a las personas actuar con idoneidad indistintamente sea la situación o el contexto en donde se encuentre. No se trata de un aprendizaje definido para un escenario en particular. Son las rutas alternativas que posibilitan a la persona acudir a sus conocimientos, buscar, detectar y seleccionar los que necesita o construir nuevos conocimientos cuando se requieren.

Contribuir al desarrollo personal y social: La competencia logra dinamizar aprendizajes que benefician a la persona, al entorno y a su comunidad, en una ruptura del individualismo y el egocentrismo que permite, desde el enriquecimiento personal, el crecimiento de los otros con los otros.

En este mismo sentido y desde los aportes de Tobón (2012) y AlemánVargas (2016) los componentes de una competencia son: 
Tabla 2. Componentes de la competencia

\begin{tabular}{ll}
\hline Identificación de la competencia & Nombre de la competencia \\
\hline Descripción de la competencia & $\begin{array}{l}\text { Redacción de la competencia, incluye } \\
\text { una acción, un objeto y una condición de } \\
\text { calidad }\end{array}$ \\
\hline Niveles de la competencia & $\begin{array}{l}\text { Grado de complejidad de acuerdo las } \\
\text { características de formación y desarrollo }\end{array}$ \\
\hline Indicador & $\begin{array}{l}\text { Desenvolvimientos o actuaciones } \\
\text { esperados }\end{array}$ \\
\hline Saberes esenciales & Saber/aprender, ser, convivir, hacer, estar \\
\hline Descriptor & $\begin{array}{l}\text { Evidencias o indicios que proveen } \\
\text { información sobre el alcance de los } \\
\text { desempeños }\end{array}$ \\
\hline
\end{tabular}

Estos componentes de la competencia deben ser sólidamente complementados desde el diseño curricular con un modelo pedagógico que defina el enfoque de las competencias, unidades de contenido que le otorguen sentido a los saberes esenciales; secuencias didácticas que permitan desarrollar de manera articulada los niveles de competencia; así como, estrategias metodológicas integradoras que permitan la apropiación y construcción experiencial de la competencia en ambientes de aprendizaje adecuados y oportunos; finalmente, un modelo de evaluación formativa construido a partir de los indicadores y descriptores de la competencia.

\section{Sobre las competencias docentes}

Así como en la definición de la competencia encontramos diversidad de enfoques y tendencias, igualmente son diversas las propuestas de autores y organizaciones que exponen cuales deberían ser las competencias del docente bajo este enfoque pedagógico. En este sentido, se plantea un ejercicio de integración de las propuestas en cinco aspectos importantes, que se muestran en el siguiente gráfico. 
Figura 1. Síntesis de las competencias docentes

Apropiación de los recursos tecnológicos

Relación asertiva con los estudiantes, pares e institución

Disposición para

el aprendizaje

Innovación y creatividad

Habilidades didácticas
Los avances en la tecnologia implican la incorporación de nuevos roles en la educación y cambios sustanciales para los docentes, quienes no pueden estar ajenos al crecimiento tecnologico y al impacto que tiene en los estudiantes de hoy.

Agrupa las competencias relacionadas con el acompañamiento en el desarrollo de aprendizajes constructivos con los estudiantes y su posición como docente mediador. De la misma manera, su compromiso institucional, la participación consensuada y cooperativa en las desiciones académicas y curriculares con sus pares y de la mima manera el compromiso ético.

Se trata de un docente que reflexiona permanentemente sobre sus prácticas y sobre esta base se capacita y se actualiza no solamente en su campo disciplinar, sino tambien en el pedagógico y la tecnología. Es un docente investigador.

Busca alternativas diferentes e innovadoras para sus prácticas pedagógicas, hace un uso creativo de los recursos disponibles para el aprendizaje y rompe los esquemas tradicionales de la educación.

Diseña ambientes de aprendizaje, tiene el dominio teorico y didactico de su disciplina. Utiliza los recursos, técnicas y estrategias de forma diversa y adecuada a los propositos de formación. Organiza y planea las rutas de aprendizaje para sus estudiantes 


\section{Propuesta de una ruta curricular de las competencias en el ámbito escolar}

Para algunas instituciones educativas resulta complejo asumir un enfoque basado en competencias. Esto se puede atribuir entre otros aspectos a que la escuela intenta implementar este proceso sin tener los fundamentos suficientes y sin desarrollar la ruta de formación docente apropiada para su comprensión, pues se piensa primero en las competencias que debe alcanzar el estudiante, sin considerar las competencias que debe tener el docente. En este sentido, se generan oposiciones o simplemente se acepta el enfoque, pero las prácticas siguen los esquemas tradicionales de la enseñanza. Otra situación recurrente, es que la institución educativa propone demasiadas competencias dificultando los procesos didácticos y la evaluación.

Una propuesta debe incluir, en primera instancia, la generación de los procesos de formación suficientes y apropiados sobre los fundamentos de la educación basada en competencias. Luego, en un ejercicio democrático de construcción pedagógica, se debe incluir el diseño de las competencias que la institución educativa considera pertinente en sus propósitos de formación, teniendo en cuenta los requerimientos que exigen las políticas nacionales y el contexto nacional e internacional para un óptimo desarrollo. En una formación por ciclos, como la propuesta por la Secretaría de Educación de Bogotá, la ruta de las competencias debería presentarse así:

En este proceso, la competencia se establece en el consenso institucional, se define para cada ciclo el nivel de competencia de acuerdo con el grado de formación. Para cada nivel de competencia, se establecen los saberes esenciales que se constituyen también en agentes generadores de contenidos para cada campo del conocimiento. De igual manera, los indicadores y descriptores constituyen la base para el modelo de evaluación institucional. 
Figura 2. Ruta de la competencia en una formación por ciclos

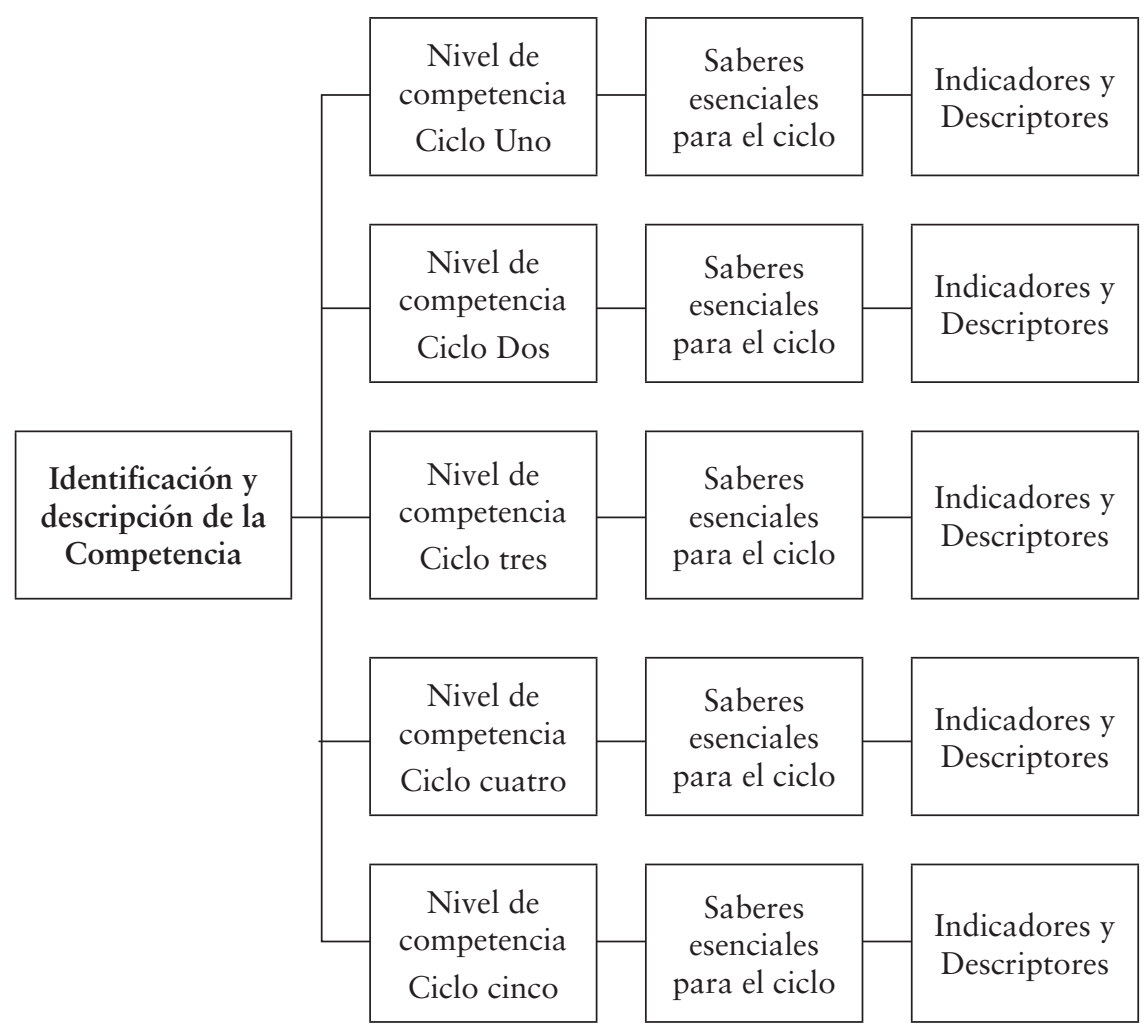

\section{Algunas conclusiones y reflexiones}

Cada uno de los enfoques que intentan definir las competencias tienen aspectos en común. Sin embargo, desde el pensamiento complejo se parte de una estructura sistémica, mucho más elaborada y con un enfoque más integral: asumen la construcción de saberes como procesos de interacción y además multidimensionales; y consideran los avances tecnológicos como generadores de cambio, obligando a transformaciones y ajustes en las dinámicas de enseñanza y aprendizaje. 
Las competencias son multidimensionales, comprometen éticamente a la persona, implican la integración de saberes y la puesta en acción de estos en escenarios reales, situados, y la capacidad de dar respuesta en diversos contextos.

Un enfoque basado en competencias no puede solucionar todos los problemas educativos ni descarta las posibilidades que ofrecen los diferentes enfoques y modelos pedagógicos. En este sentido, antes de formar en competencias a los estudiantes, se debe pensar en un docente que se haya apropiado del enfoque y posea las herramientas y habilidades para su desarrollo. El docente, más que enseñar, debe estar dispuesto a aprender, a reflexionar constantemente sobre su práctica y a superar coherentemente las dificultades en el proceso.

\section{Referencias}

Alemán Vargas, M. (2016). Fundamentos de la educación basada en competencias. Curso en: Doctorado Educación Basada en Comptencias. Centro Universitario Mar de Cortes. México.

Barón Birchenall, L. (2014). La Teoría Lingüística de Noam Chomsky: del Inicio a la Actualidad. Recuperado de http://www.scielo.org.co/pdf/leng/v42n2/ v42n2a08.pdf

Proyecto Tuning América Latina. (2007). Reflexiones y perspectivas de la educación superior en América Latina.

Tobón, S. (2008). Formación Basada en competencias en la educación superior. Instituto CIFE. Bogotá, Colombia.

Tobón, S. (2012). Formación Basada en competencias. Ecoe. Bogotá, Colombia.

Zabala, A. \& Arnau, L. (2008). 11 Ideas Clave: Cómo aprender y enseñar competencias. Graó. Barcelona, España.

Zabalza, M. A. (2003). Competencias docentes. Universidad de Santiago de Compostela. España. 